BMJ Open Sport \& Exercise Medicine

\section{Patterns of endogenous and exogenous ovarian hormone modulation on recovery metrics across the menstrual cycle}

To cite: Sims ST, Ware L, Capodilupo ER. Patterns of endogenous and exogenous ovarian hormone modulation on recovery metrics across the menstrual cycle. BMJ Open Sport \& Exercise Medicine 2021;7:e001047. doi:10.1136/ bmjsem-2021-001047

Accepted 5 July 2021
Check for updates

(c) Author(s) (or their employer(s)) 2021. Re-use permitted under CC BY-NC. No commercial re-use. See rights and permissions. Published by BMJ.

${ }^{1}$ SPRINZ, Auckland University of Technology, Auckland, New Zealand

${ }^{2}$ Te Huataki Waiora School of Health, University of Waikato, Mount Maunganui, Waikato, New Zealand

${ }^{3}$ Whoop Inc, Boston,

Massachusetts, USA

Correspondence to Dr Stacy T Sims;

stacy.sims@waikato.ac.nz

\section{ABSTRACT}

Introduction As the number of female athletes competing rises globally, training methodologies should reflect sex differences across critical metrics of adaptation to training. Surrogate markers of the autonomic nervous system (ANS) used for monitoring training load are heart rate variability $(\mathrm{HRV})$ and resting heart rate $(\mathrm{RHR})$. The aim was to investigate ovarian hormone effects on standard recovery metrics (HRV, RHR, respiratory rate (RR) and sleep duration) across a large population of female athletes.

Methods A retrospective study analysed 362852 days of data representing 13535 menstrual cycles (MC) from 4594 respondents (natural $M C n=3870, B C n=455$, progestinonly $n=269$ ) for relationships and/or differences between endogenous and exogenous ovarian hormones on ANS.

Results HRV and return to baseline (recovery) decreased as resting $H R$ and $R R$ increased $(p<0.001)$ from the early follicular to the late luteal phase of the MC. Patterning was paradoxical across phases for users of combined hormonal contraception $(\mathrm{BC})$ as compared with the patterning of the MC. HRV and recovery start elevated and drop off quickly during the withdrawal bleed, rising through the active pill weeks $(p<0.001)$. Progestin-only users had similar patterning as the MC. The relationship between normalised recovery and previous day strain is modulated by birth control type. BC exhibited steeper declines in recovery with additional strain-normalised recovery decreases by an additional $0.0055 \pm 0.00135(p<0.001)$ per unit of strain; with no significant difference between MC and progestinonly $(p=0.19)$.

Conclusion The patterning of ANS modulation from ovarian hormones is significantly different between naturally cycling women and those on $\mathrm{BC}$, with the patterning dependent on the type of contraception used.

\section{INTRODUCTION}

The female hormonal cycle and its systemic effects on the body is a crucial area for research as it has been found that women frequently experience different adaptations and stress responses to their male counterparts, at times as a result of female hormonal fluctuations. ${ }^{1}$ The menstrual cycle (MC) is characterised by fluctuations in several hormones, most notably the gonadal steroids, oestrogen and

\section{Key messages}

What are the new findings

- Ovarian hormones modulate standard recovery metrics (respiratory rate, sleep measures) used to determine adaptation to training load and stress across the menstrual cycle.

- Indices of lower recovery and higher strain are associated with the luteal phase of the natural menstrual cycle due to progesterone stimulatory influence on the autonomic nervous system (ANS).

- Exogenous hormones of birth control (BC) modulate the recovery metrics in a different pattern than the natural menstrual cycle, with reduced indices of adaptation to stress across all pill phases.

- Exogenous hormone use significantly impacts day to day recovery to increased cardiovascular strain as compared with the natural menstrual cycle.

- Progestin-only BC exhibit a unique modulation of recovery indices, with a lessened effect on the ANS in the late luteal phase.

- Progestin-only BC exhibit similar patterns to the natural menstrual cycle with regard to cardiovascular strain and next day recovery.

How might it impact on clinical practice in the future

- Current practices in sport and exercise training programmes do not account for the influence of ovarian hormones on the ANS, nor are the differences between endogenous and exogenous hormone profiles acknowledged in coaching and exercise prescription. By understanding the modulatory effect exerted by the different hormone profiles, improved training methodologies may be employed to improve the adaptations to training loads of female athletes.

progesterone, and is divided into four main hormonal phases, the early follicular, midfollicular, early luteal and late-luteal phases. Throughout each phase, fluctuations in hormones trigger not only changes in the reproductive system, but also in all the tissues of the body, ${ }^{1}$ modulating sleep, ${ }^{23}$ circadian rhythms ${ }^{4}$ and the autonomic nervous system (ANS) ${ }^{5-7}$ For instance, there is evidence of a 
decrease of the vagal dominance on the heart from the follicular to the luteal phase. ${ }^{6-8}$ Moreover, baroreflex sensitivity (BRS) is higher in the luteal phase of the cycle, presumably due to the elevation of progesterone and its stimulation of the phrenic nerve and overall sympathetic drive. ${ }^{68}$

A surrogate marker for ANS activity is heart rate variability (HRV), the recording of in-between heartbeat variations that are modulated by parasympathetic and sympathetic inputs. ${ }^{9-11} \mathrm{HRV}$ is sensitive to homoeostatic perturbations like fatigue, physiological and psychological stress. ${ }^{89}$ Across sports, HRV is widely used as an appropriate criterion for training load quantification, ${ }^{9}$ as the combined intensity and duration of a training session impacts the magnitude and time required for recovery from the induced stress response, shifting the autonomic balance. Periods of higher cumulative stress are typically highlighted by a reduction in HRV and in situations of positive adaptations to training, HRV should increase or remain stable, with increasing training loads. Thus, resting heart rate (RHR) and HRV have been used to adjust training loads and identify positive and negative adaptations to aerobic training. ${ }^{1011}$

As an important marker for quantifying adaptation to training load, there is a lack of attention to how female sex hormones modulate ANS activity within exercise training/prescription, coaching and applied sport science, ${ }^{12} 13$ impacting quantification and monitoring training loads specific to female athletes. In addition, regardless of sport, there is a high percentage of female athletes who use hormonal contraception.${ }^{14}$ Combined oral contraceptives (COC) contain derivatives of oestrogens and progestogens, which downregulate the regular cyclic hormone activity of the hypothalamic-pituitarygonadal axis, resulting in physiological responses which differ to those of endogenous ovarian hormones. ${ }^{15}$ For example, Wilczak et $a l^{16}$ compared indices of the ANS between women using COC and naturally cycling women, finding a downregulation of BRS with COC use. Moreover, Teixeira $e t a l^{7}$ determined COC do not affect HRV across the active pill phases of use.

The aim of this project was to investigate ovarian hormone effects on standard recovery metrics of HRV, RHR, respiratory rate (RR) and sleep duration across a large population of female athletes, and to determine any differences between endogenous and exogenous hormones on these metrics.

\section{METHODS}

Data were collected retrospectively from subscribers to the WHOOP, Inc. platform. Specifically, RHR, HRV, RR and the percentage of time in the four sleep stages (wake, light sleep, REM and slow wave sleep) were captured using a wrist-worn multisensor (triaxial accelerometer, optical sensor, capacitive touch sensor and ambient temperature sensor) device (WHOOP, Inc., Boston, Massachusetts USA). Participant characteristics, including age, weight, height and athletic status (elite or recreational), were determined through mandatory in-app questions that must be completed during the sign-up process. Data for the current study were analysed from an optional survey of women who recorded menstruation on the app from 29 October 2020 to 9 December 2020. Inclusion criteria included regular, natural MCs lasting between 25 and 35 days, or the use of hormonal contraceptives (including the inactive pill week). Exclusion criteria included: irregular cycles (as defined as missing two or more cycles in a 6-month period or shorter than 25 days or longer than 35 days), uncertainty of the type of birth control (BC) used, and/or having stopped using BC within the past 9 months. All participants in the study had consented at registration to the use of their data for the purposes of scientific research and could withdraw consent at any time.

Measures of RHR and HRV were evaluated during the last slow wave cycle of sleep, which was shown to be the best recording condition because of a high level of environmental and respiratory standardisation. ${ }^{18}{ }^{19} \mathrm{RR}$ was calculated as the median RR over the entire sleep period. RHR, HRV and RR are derived via photoplethysmography recordings at the wrist, which has been demonstrated to be a valid method for measurement of R-R intervals via ECG while at rest. ${ }^{20-22}$ Recovery is a proprietary metric of the WHOOP, Inc. platform that combines nighttime measures of heart rate, HRV, RR and sleep duration into a once-daily metric used to guide recommended training load. ${ }^{23}$ Recovery ranges from $0 \%$ to $100 \%$ with higher values indicating greater readiness to adapt to a training load. All measures have been previously validated in female athletes as a means to quantify training load and training stress. ${ }^{123-26}$

The WHOOP data are the property of WHOOP, Inc. The authors (LW and ERC) are employees of WHOOP, Inc and are permitted to access the WHOOP data server.

\section{Statistical analysis}

This study's primary interest was the effect of menstrual phase on physiological measures of recovery and the modulation of endogenous and exogenous hormones on these effects. Daily measures of HRV, resting HR, RR and recovery were analysed for the study population. The menstrual phase for each individual and day was estimated by taking the corresponding $\mathrm{MC}$ and dividing it into four equally spaced intervals with the first day of self-reported menstruation defining the beginning of the early follicular phase. Days that fell in the first $25 \%$ of the cycle were designated as early follicular phase. The next $25 \%$ interval was designated as late follicular phase. The next $25 \%$ was designated as early luteal phase, and the last $25 \%$ was designated as late luteal phase. Days corresponding to the withdrawal bleed of the $\mathrm{BC}$ were designated as the early follicular phase, with the subsequent active pill weeks designated as 'week 2 , week 3 , week 4' to correspond with the natural cycle 'late follicular, early luteal, late luteal' phases. All statistical analyses were performed in R (V.4.0.3). 
Table 1 Participant characteristics

\begin{tabular}{|c|c|c|c|c|}
\hline & Total & MC & BC & Progestin-Only \\
\hline & $\mathrm{N}=4594$ & $\mathrm{~N}=3870$ & $\mathrm{~N}=455$ & $\mathrm{~N}=269$ \\
\hline Age (years) & $33.5(7.3)$ & $34.1(7.3)$ & $29.9(6.3)$ & $31.3(6.2)$ \\
\hline Weight (kg) & $67.7(12.3)$ & $67.8(12.2)$ & $65.8(11.6)$ & $69.3(13.5)$ \\
\hline Height (m) & $1.66(0.069)$ & $1.66(0.070)$ & $1.65(0.067)$ & $1.67(0.069)$ \\
\hline $\mathrm{BMI}\left(\mathrm{kg} / \mathrm{m}^{2}\right)$ & $24.5(4.1)$ & $24.5(4.2)$ & $24.0(3.8)$ & $24.7(4.3)$ \\
\hline RHR (bpm) & $57(7)$ & $57(7)$ & $59(7)$ & $58(8)$ \\
\hline Daily strain & $10.9(2.6)$ & $10.9(2.6)$ & $10.8(2.3)$ & $10.8(2.5)$ \\
\hline Total hour exercise/week* & $5.5(3.9)$ & $5.5(4.0)$ & $5.2(3.5)$ & $5.1(3.3)$ \\
\hline Moderate intensity hour/week & $2.5(2.1)$ & $2.5(2.1)$ & $2.4(2.0)$ & $2.3(1.8)$ \\
\hline Vigorous intensity hour/week & $2.0(1.6)$ & $2.0(1.6)$ & $2.0(1.6)$ & $2.0(1.7)$ \\
\hline
\end{tabular}

*Intensity as defined by the American Heart Association (AHA) ${ }^{38}$

$\mathrm{BC}$, birth control; $\mathrm{BMI}$, body mass index; ; $\mathrm{MC}$, naturally cycling; RHR, resting heart rate.

\section{Statistical analysis: MC phase and HC}

In the analysis, linear models were used to relate each physiological measure (normalised HRV, normalised RR, normalised resting HR, normalised recovery score) to menstrual phase and BC type. Interaction effects were included in the models. Extra sum-of-squares F-tests were performed to test for the significance of the main effects and interaction effects. For hypothesis tests with multiple comparisons, a Bonferroni correction was applied to the alpha level of 0.05 to generate familywise alpha levels.

\section{Statistical analysis: cardiovascular strain and recovery}

Previous day cardiovascular strain was added to the existing model, including interactions with menstrual phase and with BC category. Extra sum-of-squares F-tests were performed to test for the significance of the main effect of cardiovascular strain and the interaction terms.

\section{RESULTS}

The final dataset included 362852 days of data representing 13535 MCs $(2.9 \pm 2.0$ cycles per participant; $28.4 \pm 2.4$ days per cycle) from 4594 respondents (natural MC $n=3870, B C n=455$, progestin-only $n=269$ ). The participants were $33.5( \pm 7.3)$ years, with a body mass index of $24.5( \pm 4.1)$, exercising 5.6 (4.1) hours per week, with a daily strain of $10.9( \pm 2.6)$ (table 1$)$.

Natural MC versus BC: HRV and recovery decreased across the MC from the mid-follicular phase whereas resting HR and RR increased. The effect of menstrual phase was found to be statistically significant at an alpha level of $0.0125(\mathrm{p}<0.001)$ for all measures. The patterning of these physiological measures is paradoxical across phases for those individuals using BC. HRV and recovery start elevated and drop off quickly during the withdrawal bleed (inactive pill week), rising through the active pill weeks (corresponding 'week 2: late follicular', 'week 3: early luteal' and 'week 4: late luteal' phases). Resting HR starts low during the first few days of the withdrawal bleed (inactive pill week) and quickly rises in the later days of the bleed week, then slowly decreases across the
3 weeks of active pill use. RR dips to its lowest point in the later days of the withdrawal bleed (corresponding early follicular), then increases across the active pill weeks, reaching its peak at the end of the fourth week of the active pill (corresponding late luteal phase). The effect of menstrual phase was found to be statistically significant at an alpha level of $0.0125(\mathrm{p}<0.001)$ for all measures. The interaction between BC category (Natural MC vs BC) and menstrual phase was also found to be statistically significant for all measures at an alpha of $0.0125(\mathrm{p}<0.001)$, suggesting that the use of $\mathrm{BC}$ modulates the patterning of these physiological measures over the cycle (figures 1 and 2).

Natural MC versus Progestin only: For individuals using progestin-only $\mathrm{BC}$, the effect of menstrual phase was similarly found to be statistically significant at an alpha level of $0.0125(\mathrm{p}<0.001)$ for all measures. Again, the patterning of these measures was found to be distinct from the patterning for the natural MC group. The interaction between hormonal BC category (natural MC vs progestin-only BC) and menstrual phase was found to be statistically significant at an alpha of $0.0125(\mathrm{p}<0.001)$ for all measures (figures 3 and 4).

\section{Cardiovascular strain and next-day recovery}

There is evidence that the relationship between normalised recovery and previous day strain is modulated by BC type. Specifically, those in the HC groups exhibited steeper declines in recovery with additional strain-normalised recovery decreases by an additional $0.0055 \pm 0.00135 \quad(\mathrm{p}<0.001$, significant at an alpha of $0.025)$ per unit of strain for individuals using combination BC, and an additional $0.0025 \pm 0.0019(\mathrm{p}=0.19$, not significant at an alpha of 0.025 ) for progestin-only BC individuals (figure 5). The main effect of previous day strain was found to be statistically significant $(p<0.001$, alpha of 0.0125), the inclusion of the interaction between previous day strain and menstrual phase was found to be statistically significant $(p<0.001$, alpha of 0.0125$)$, and the inclusion of the interaction between previous day strain 

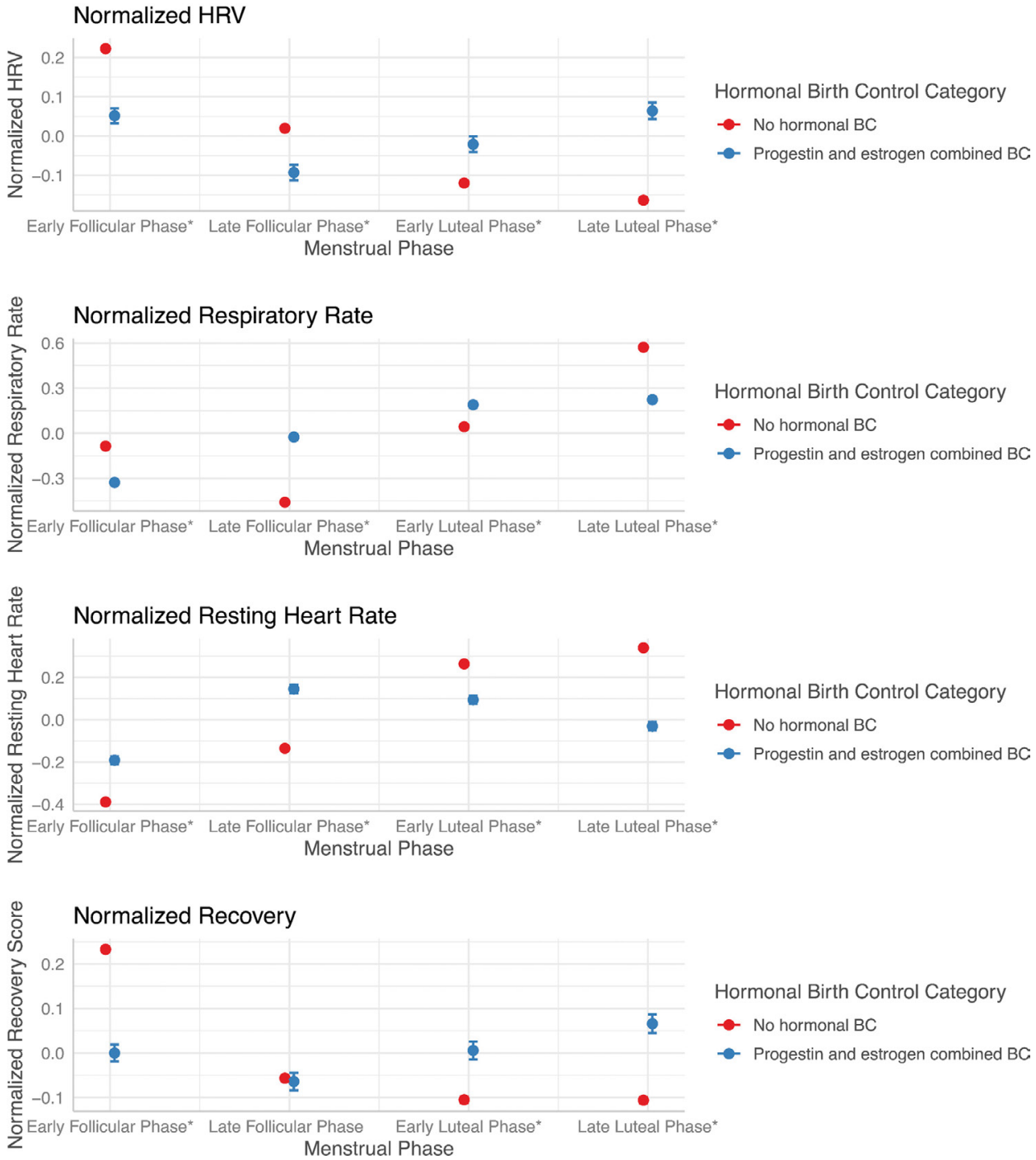

Figure 1 Effects of natural versus $B C$ menstrual phases on physiological measures. BC, birth control; HRV, heart rate variability. ${ }^{*}$ denotes significance with $\alpha$ set at $0.003125 ; p<0.001$.

and BC category was found to be statistically significant $(\mathrm{p}<0.001$, alpha of 0.0125$)$.

\section{DISCUSSION}

The ANS is essential for regulating heart rate and arterial pressure via the arterial baroreflex. The activity of the autonomic nerves is controlled centrally via nuclei which relay excitatory or inhibitory information to the preganglionic autonomic fibres in the sympathetic and/ or parasympathetic pathways. ${ }^{816}$ Sex hormones directly affect the activity of these pathways, thus altering peripheral sympathetic and parasympathetic neural activity, and subsequently, cardiovascular function. ${ }^{6} 26$

HRV analysis is a valuable, objective marker of ANS used to study an athlete's training vs recovery equilibrium and detect early overreaching state that can decrease the athlete's performance. ${ }^{11-13} 1924$ To our knowledge, existing research and its application to female athletes has been generalised from male data, despite limited research indicating significant sex differences and responses of HRV and the ANS. ${ }^{512} 13$ This generalisation is a significant misstep for female athletes as the neural control of circulation differs with sex, mainly attributed to the effects of ovarian hormones on ANS. ${ }^{8} 16232526$ Moreover, endogenous and exogenous hormones exert different modulatory effects; specific to the progestogen of the exogenous hormone formulation. ${ }^{16172627}$ The current study is the first to investigate HRV, RHR, RR and sleep metrics in a large population of female athletes; to include naturally cycling women, women using COC and women using progestin-only contraception.

\section{Endogenous versus exogenous hormone modulatory effects}

In this robust dataset, it was observed that the patterning of ANS modulation from ovarian hormones is significantly different between naturally cycling women and those on $\mathrm{BC}$; with the patterning dependent on the type of BC used.

Specifically, in naturally cycling women, HRV and recovery are elevated in the early and mid-follicular phases with a significant decline of HRV and recovery metrics into the late luteal phase. As a brief review, the 

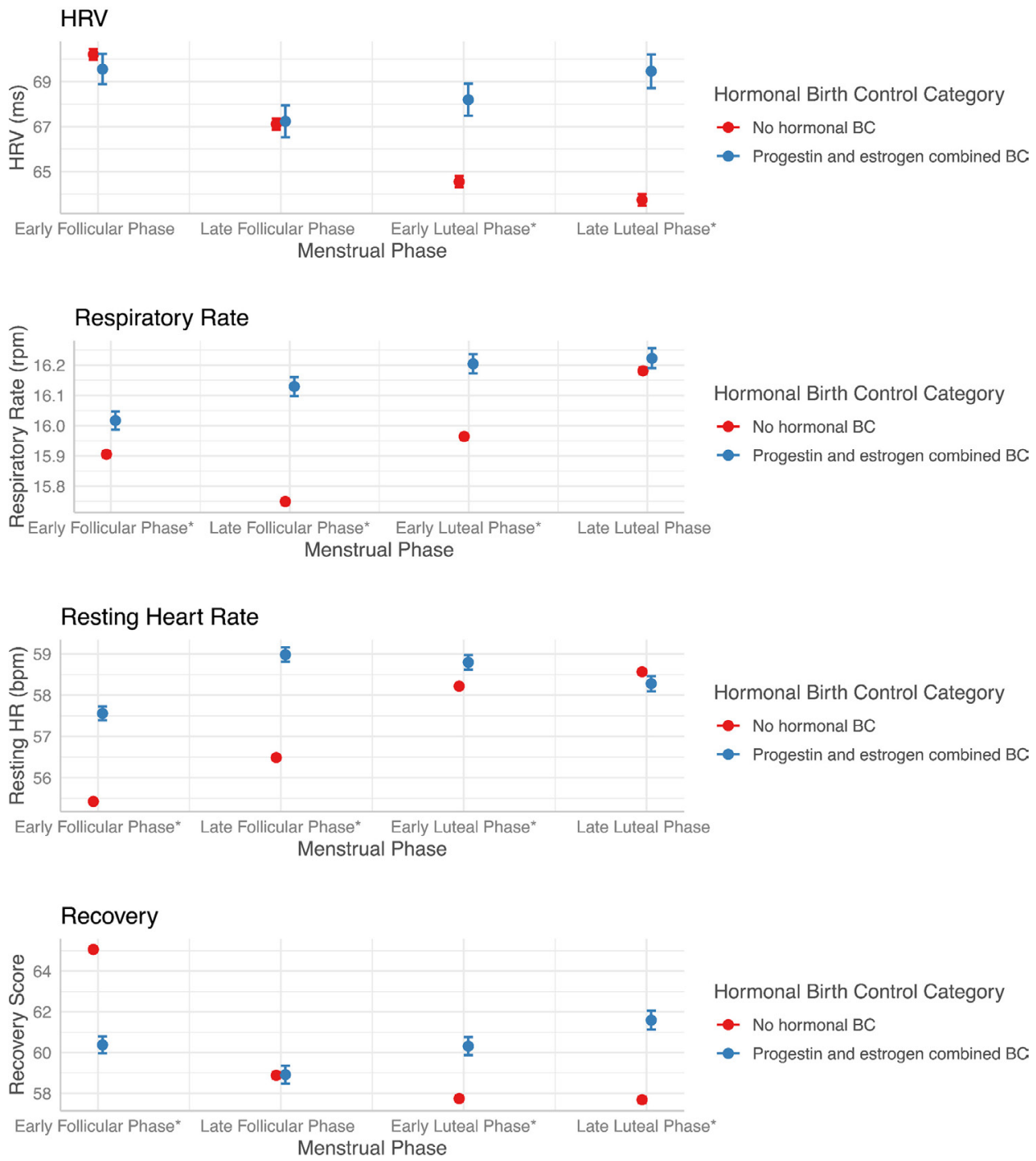

Figure 2 Effects of natural versus BC menstrual phases on unnormalised physiological measures. BC, birth control; HRV, heart rate variability. ${ }^{*}$ denotes significance with $\alpha$ set at $0.003125 ; p<0.001$.

first half of the MC comprised by the menstrual and follicular phases during which time oestrogen levels are low (menstrual phase) and rise (follicular phase) and ends with the periovulatory phase in which follicularstimulating hormone and luteinising hormones peak. The second half of the cycle is comprised by the luteal (during which time oestrogen level rises with a progesterone peak) and the premenstrual phases during which time oestrogen and progesterone levels fall (figure 6). It is known that oestrogens act centrally to modulate the autonomic nervous system, increasing vagal and decreasing sympathetic activity, whereas progesterone appears to have an opposing effect, elevating central norepinephrine release inducing a sympathetic drive. ${ }^{2427}$ Thus, due to the unopposed oestrogen in the early and mid-follicular phases, followed by the elevation of progesterone in the luteal phase which antagonises oestrogenic effects, it is unsurprising to note this pattern of HRV and recovery in naturally cycling women.

However, in women using combined BC, a different, paradoxical pattern emerges. We observed an elevated
HRV and recovery in the withdrawal phase (inactive pill phase), with a significant decline at the onset of active pill use. This patterning may be representative of the perturbation of exogenous hormones. In the withdrawal phase of BC use, exogenous hormones are not consumed, thus their effect on the downregulation of the HPA axis is removed; given the different half-lives of the exogenous steroids and variable impact on the endogenous hormones, the withdrawal week should be considered a transient hormonal profile phase. ${ }^{128}$ With resuming exogenous hormone consumption, a stable hormone profile emerges; the inhibitory effects of both the oestrogen and the progestin components are established through synergistic interactions at the hypothalamicpituitary level. Recently, Assadpour et $a l^{29}$ suggested the chronic upregulation of $\beta$-adrenergic receptors of $\mathrm{BC}$ users may play a role in the upregulation of ventilatory rate, and autonomic reflex function, which is reflected in the patterning of the women on BC of this study of decreased HRV, increased RR, increased RHR across the active pill phases. 
Normalized HRV
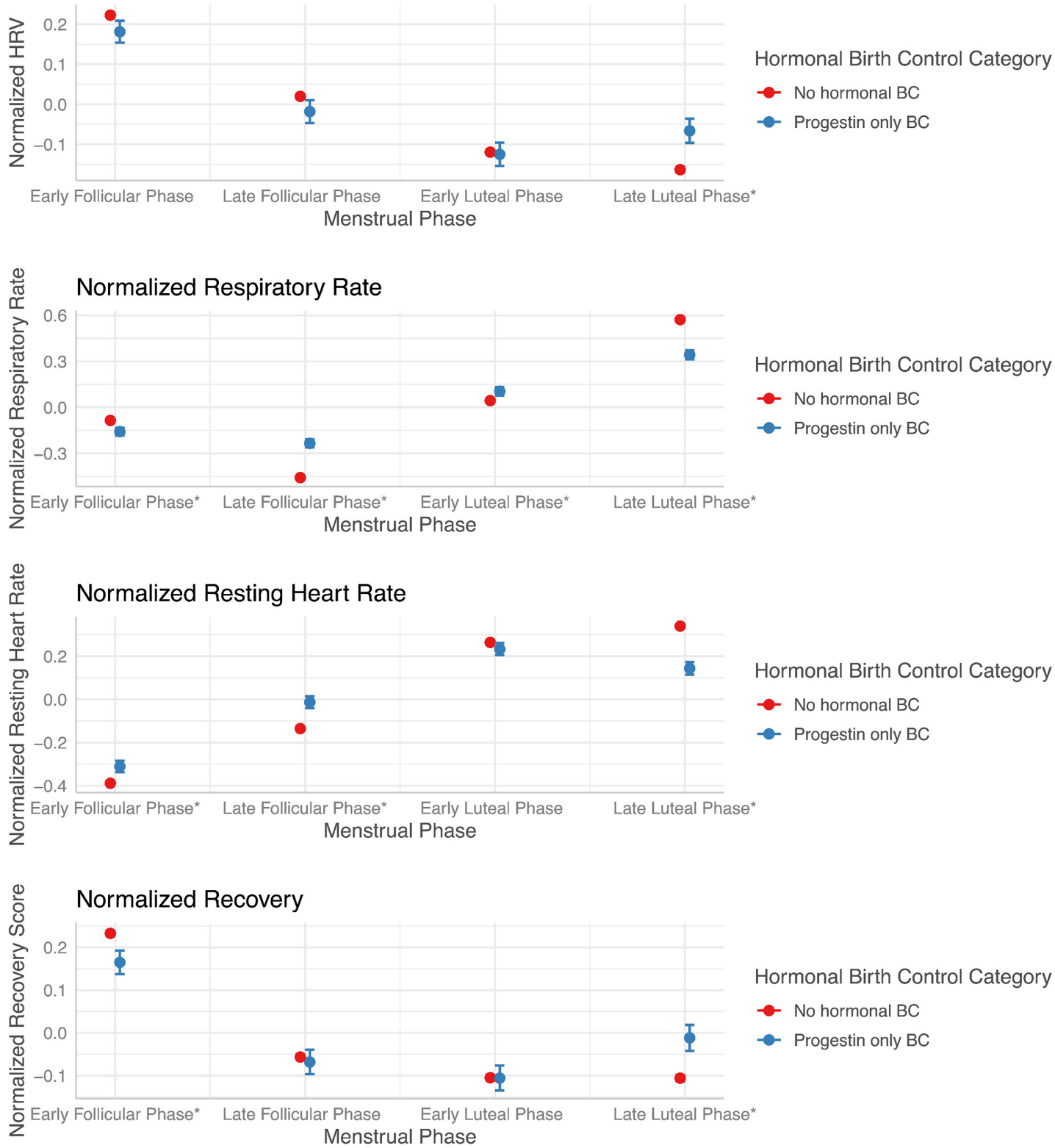

Figure 3 Effects of natural versus progestin-only menstrual phases on physiological measures. BC, birth control; HRV, heart rate variability. ${ }^{*}$ denotes significance with $\alpha$ set at $0.003125 ; p<0.001$.

Interestingly, women taking the progestin-only contraception exhibited a similar pattern as naturally cycling women except for the late luteal phase in which there was an observed attenuation of sympathetic drive. This may be due to the generation of the progestogens used in the progestin-only formulations. The progestin-only formulations use only norethisterone, a first-generation progestin, or desogestrel, a third-generation progestin, which are 1.5 times more potent than endogenous progesterone,${ }^{27}$ thus may attenuate the magnitude of the phase-based increase of sympathetic drive in the later stages of the cycle.

\section{Cardiovascular strain and next day recovery}

The application of appropriate training load is one of the fundamental factors for positive physiological adaptations to occur with improvements in performance. Monitoring training loads and recovery should reduce the incidence of excessive loading, which results in negative adaptations (including non-functional overtraining). ${ }^{30}$ 31 A major tenet in determining load application is based on cardiovascular strain; periods of higher cumulative stress are typically highlighted by a reduction in HRV and in situations of positive adaptations to training, HRV should increase, or remain stable, with increasing training loads. The data from the current study indicates a reduced recovery in women using combined BC with every unit of increased strain, as compared with naturally cycling or progestin-only pill women across regardless of cycle phase. In addition to the exogenous effects described above, recovery may have been attenuated due to greater 

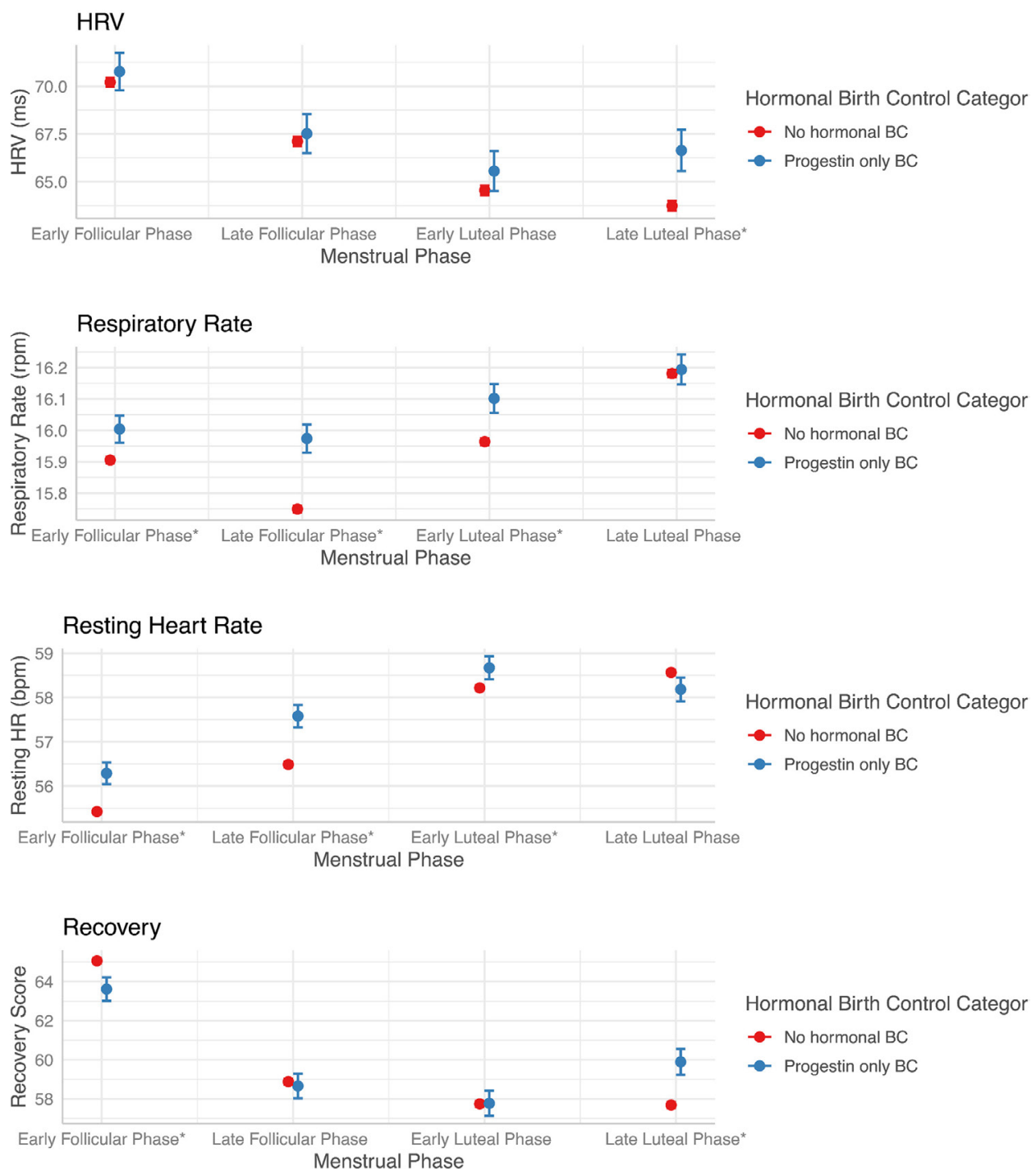

Figure 4 Effects of natural versus progestin-only BC menstrual phases on unnormalised physiological measures. BC, birth control; HRV, heart rate variability. *denotes significance with $\alpha$ set at $0.003125 ; p<0.001$.

inflammatory responses associated with $\mathrm{BC}$ use $\mathrm{e}^{32-34}$ and to the difference in stress responsiveness of women on BC. Larsen $e t a e^{34}$ reported a higher incidence of IL-6 and $\mathrm{C}$ reactive protein (CRP) in Olympic-calibre female athletes using $\mathrm{BC}$ as compared with the same calibre naturally cycling female athlete. Moreover, Cauci et $a \vec{l}^{33}$ observed a marked elevation of low-grade elevation (as measured by CRP) in female athletes using BC, which may exacerbate inflammatory responses to physical stress, increasing the time course for recovery. Of greater interest as it pertains to the ANS is the difference between naturally cycling and BC users free cortisol. It

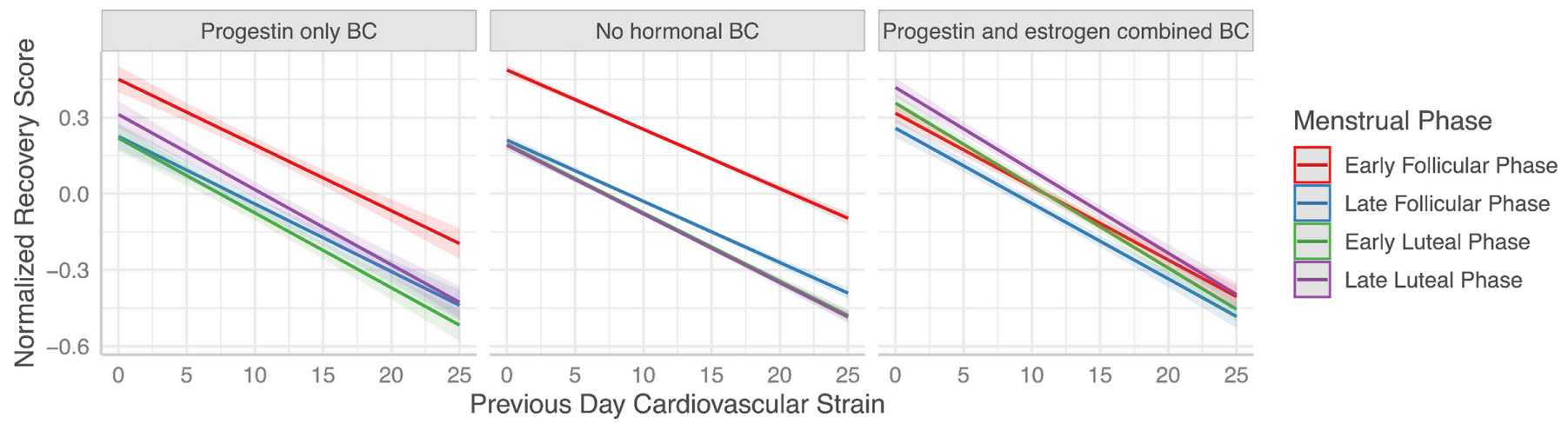

Figure 5 Modulation of cardiovascular strain and next day recovery by hormonal contraception. BC, birth control. 

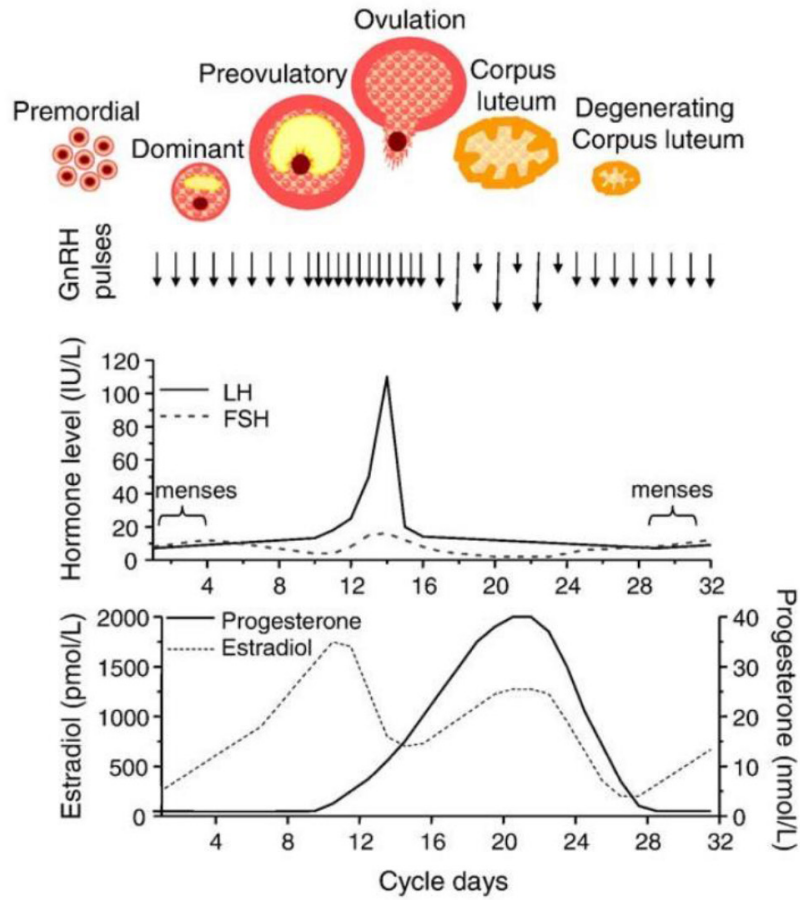

Figure 6 Diagram of the menstrual cycle. FSH, follicularstimulating hormone; LH, luteinising hormone.

has been repeatedly shown that $\mathrm{BC}$ increase free circulating cortisol in the blood ${ }^{35} 36$ which is associated with chronically elevated cortisol levels and a reduced diurnal rhythm. ${ }^{37}$ As cortisol is a known stimulatory hormone, increasing the threshold for parasympathetic drive, this may be a mechanism responsible for decreased recovery metrics per unit of strain.

\section{Limitations and strengths}

Strengths of this project include the large dataset of physically active premenopausal women; to include normally cycling, COC users, and progestin-only pill users; allowing for robust comparisons which have not been identified in previous literature to the authors knowledge.

This project has some limitations inherent to the data retrieved. One limitation is the classification of the $\mathrm{MC}$ phase. Determination of the MC phase was somewhat uncertain as the model assumed fertility in a regular 28-day cycle, which may not correspond to the phases determined by modern hormonal documentation or the presence of anovulatory cycles. As this was a large retrospective study, MC phases were not confirmed with blood tests (as is the gold standard, ${ }^{1}$ which does not allow for identification of extended follicular phases or luteal phase deficiencies. Second, identification of the generation of the progestin in the oral contraceptive pills was not feasible except for the progestin-only (as there are only two progestins available to market for the minipill). The mechanism of progestins is a multimodal one, and each subsequent generation of progestogen has a different binding affinity to steroid receptors and serum binding proteins ${ }^{27}$ which may affect the threshold of parasympathetic downregulation. Third, due to privacy and deidentification of data, specific identifiers such as ethnicity, socioeconomic status, medication usage, cardiovascular and metabolic disease risk factors and other dietary-lifestyle choices were not included in the data aggregation and analysis. Further research lends to the investigation of these different progestin generations on parasympathetic thresholds and vagal tone. To note, a unique limitation, in this era of COVID-19, is the psychological stress of the pandemic which may have influenced HRV and recovery metrics.

\section{CONCLUSION}

As the number of female athletes competing across all levels rises, training methodologies should evolve to reflect sex differences across critical metrics of adaptation to training. Using a robust data set (13535 MCs from 4594 respondents) we presented novel data with regards to recovery metrics across three different hormone profiles of female athletes: naturally cycling, COC and progestin-only users. Understanding how endogenous and exogenous hormones modulate the autonomous nervous system and subsequent recovery metrics is critical for monitoring training loads and the application of appropriate training stress.

Contributors STS led the work of the writing group and wrote the manuscript. ERC and LW analysed the data, contributed to the writing and editing. All authors contributed to the design of the study, interpreted the data and critically reviewed the report.

Funding The authors have not declared a specific grant for this research from any funding agency in the public, commercial or not-for-profit sectors.

Competing interests Two of the authors (ERC, LW) are affiliated to the commercial company WHOOP, however, this does not alter the authors' adherence to BJSM policies on sharing data and materials.

Patient and public involvement Patients and/or the public were involved in the design, or conduct, or reporting, or dissemination plans of this research. Refer to the Methods section for further details.

Patient consent for publication Not required.

Ethics approval The HREC board that governs WHOOP data exempted this study in line with the NIH ruling of exempt data (aggregated and deidentified).

Provenance and peer review Not commissioned; externally peer reviewed.

Data availability statement Data are available on reasonable request. The WHOOP data are the property of WHOOP (Boston, Massachusetts, USA). The authors (LW and ERC) are employees of WHOOP and are permitted to access the WHOOP data server. The data are anonymised appropriately; the data used in the study is a backup of the original data and does not include any personally identifiable information.

Open access This is an open access article distributed in accordance with the Creative Commons Attribution Non Commercial (CC BY-NC 4.0) license, which permits others to distribute, remix, adapt, build upon this work non-commercially, and license their derivative works on different terms, provided the original work is properly cited, appropriate credit is given, any changes made indicated, and the use is non-commercial. See: http://creativecommons.org/licenses/by-nc/4.0/.

ORCID iD

Stacy T Sims http://orcid.org/0000-0002-3255-5428

\section{REFERENCES}

1 Sims ST, Heather AK, Myths HAK. Myths and methodologies: reducing scientific design ambiguity in studies comparing sexes and/or menstrual cycle phases. Exp Physiol 2018;103:1309-17. 
2 Zhang S, Osumi H, Uchizawa A, et al. Changes in sleeping energy metabolism and thermoregulation during menstrual cycle. Physiol Rep 2020;8:e14353.

3 Baker FC, Lee KA. Menstrual cycle effects on sleep. Sleep Med Clin 2018;13:283-94.

4 Baker FC, Driver HS. Circadian rhythms, sleep, and the menstrual cycle. Sleep Med 2007;8:613-22.

5 Bassareo PP, Crisafulli A. Gender differences in hemodynamic regulation and cardiovascular adaptations to dynamic exercise. Curr Cardiol Rev 2020;16:65-72.

6 Brooks VL, Cassaglia PA, Zhao D, et al. Baroreflex function in females: changes with the reproductive cycle and pregnancy. Gend Med 2012;9:61-7.

7 de Zambotti M, Nicholas CL, Colrain IM, et al. Autonomic regulation across phases of the menstrual cycle and sleep stages in women with premenstrual syndrome and healthy controls. Psychoneuroendocrinology 2013;38:2618-27.

8 Tanaka M, Sato M, Umehara S, et al. Influence of menstrual cycle on baroreflex control of heart rate: comparison with male volunteers. Am J Physiol Regul Integr Comp Physiol 2003;285:R1091-7.

9 Kiviniemi AM, Hautala AJ, Kinnunen $\mathrm{H}$, et al. Endurance training guided individually by daily heart rate variability measurements. Eur J Appl Physiol 2007;101:743-51.

10 Buchheit M. Monitoring training status with HR measures: do all roads lead to Rome? Front Physiol 2014;5:73.

11 Plews DJ, Laursen PB, Kilding AE, et al. Evaluating training adaptation with heart-rate measures: a methodological comparison. Int J Sports Physiol Perform 2013;8:688-91.

12 Schäfer D, Gjerdalen GF, Solberg EE, et al. Sex differences in heart rate variability: a longitudinal study in international elite crosscountry skiers. Eur J Appl Physiol 2015;115:2107-14.

13 da Silva VP, de Oliveira NA, Silveira $\mathrm{H}$, et al. Heart rate variability indexes as a marker of chronic adaptation in athletes: a systematic review. Ann Noninvasive Electrocardiol 2015;20:108-18.

14 Martin D, Sale C, Cooper SB, et al. Period prevalence and perceived side effects of hormonal contraceptive use and the menstrual cycle in elite athletes. Int J Sports Physiol Perform 2018;13:926-32.

15 Verrilli LE, Landry M, Blanchard H. Contraceptive choices and menstrual patterns in high level female athletes. Fertility and Sterility 2017;108:e122.

16 Wilczak A, Marciniak K, Kłapciński M, et al. Relations between combined oral contraceptive therapy and indices of autonomic balance (baroreflex sensitivity and heart rate variability) in young healthy women. Ginekol Pol 2013;84:915-21.

17 Teixeira AL, Ramos PS, Vianna LC, et al. Heart rate variability across the menstrual cycle in young women taking oral contraceptives. Psychophysiology 2015;52:1451-5.

18 Achten J, Jeukendrup AE. Heart rate monitoring: applications and limitations. Sports Med 2003;33:517-38.

19 Buchheit M, Simon C, Charloux A, et al. Relationship between very high physical activity energy expenditure, heart rate variability and self-estimate of health status in middle-aged individuals. Int J Sports Med 2006;27:697-701.

20 Jeyhani V, Mahdiani S, Peltokangas M, et al. Comparison of HRV parameters derived from photoplethysmography and electrocardiography signals. Annu Int Conf IEEE Eng Med Biol Soc 2015;2015:5952-5.
21 Lu G, Yang F, Taylor JA, et al. A comparison of photoplethysmography and ECG recording to analyse heart rate variability in healthy subjects. J Med Eng Technol 2009;33:634-41.

22 Pinheiro N, Couceiro R, Henriques J, et al. Can PPG be used for HRV analysis? Annu Int Conf IEEE Eng Med Biol Soc 2016;2016:2945-9.

23 Sekiguchi Y, Adams WM, Benjamin CL, et al. Relationships between resting heart rate, heart rate variability and sleep characteristics among female collegiate cross-country athletes. J Sleep Res 2019;28:e12836.

24 Hautala AJ, Kiviniemi AM, Tulppo MP. Individual responses to aerobic exercise: the role of the autonomic nervous system. Neurosci Biobehav Rev 2009;33:107-15.

25 Tenan MS, Brothers RM, Tweedell AJ, et al. Changes in resting heart rate variability across the menstrual cycle. Psychophysiology 2014;51:996-1004.

26 von Holzen JJ, Capaldo G, Wilhelm M, et al. Impact of endo- and exogenous estrogens on heart rate variability in women: a review. Climacteric 2016;19:222-8.

27 Regidor P-A. The clinical relevance of progestogens in hormonal contraception: present status and future developments. Oncotarget 2018;9:34628-38.

28 Rible RD, Taylor D, Wilson ML, et al. Follicular development in a 7-day versus 4-day hormone-free interval with an oral contraceptive containing $20 \mathrm{Mcg}$ ethinyl estradiol and $1 \mathrm{Mg}$ norethindrone acetate. Contraception 2009;79:182-8.

29 Assadpour E, Ivry I, Wasef S, et al. Oral contraceptives and menstrual cycle influence autonomic reflex function. Physiol Rep 2020;8:e14550.

30 Gabbett TJ. Debunking the myths about training load, injury and performance: empirical evidence, hot topics and recommendations for practitioners. Br J Sports Med 2020;54:58-66.

31 Gabbett TJ. The training-injury prevention paradox: should athletes be training smarter and harder? Br J Sports Med 2016;50:273-80.

32 Dreon DM, Slavin JL, Phinney SD. Oral contraceptive use and increased plasma concentration of C-reactive protein. Life Sci 2003;73:1245-52.

33 Cauci S, Francescato MP, Curcio F. Combined oral contraceptives increase high-sensitivity C-reactive protein but not haptoglobin in female athletes. Sports Med 2017;47:175-85.

34 Larsen B, Cox A, Colbey C, et al. Inflammation and ora contraceptive use in female athletes before the Rio Olympic Games. Front Physiol 2020;11:497.

35 Meulenberg PM, Ross HA, Swinkels LM, et al. The effect of oral contraceptives on plasma-free and salivary cortisol and cortisone. Clin Chim Acta 1987;165:379-85.

36 McQuaid RJ, McInnis OA, Paric A, et al. Relations between plasma oxytocin and cortisol: the stress buffering role of social support. Neurobiol Stress 2016;3:52-60.

37 Miller GE, Chen E, Zhou ES. If it goes up, must it come down? Chronic stress and the hypothalamic-pituitary-adrenocortical axis in humans. Psychol Bull 2007;133:25-45.

38 Norton K, Norton L, Sadgrove D. Position statement on physical activity and exercise intensity terminology. J Sci Med Sport 2010;13:496-502. 